\title{
Distinguishing benign from malignant parotid gland tumours: low-dose multi-phasic CT protocol with 5-minute delay
}

\author{
G. Q. Jin • D. K. Su • D. Xie • W. Zhao • L. D. Liu • \\ X. N. Zhu
}

Received: 12 November 2010 /Revised: 12 February 2011 /Accepted: 18 February 2011 /Published online: 11 March 2011

(C) The Author(s) 2011. This article is published with open access at Springerlink.com

\begin{abstract}
Objectives To explore the percentage enhancement washout ratio (PEW) and relative PEW (RPEW) of low-dose multi-phasic computed tomography (CT) in distinguishing benign from malignant parotid gland tumours.

Methods This study was approved by the ethics committee, and informed patient consent was obtained. 51 patients with parotid tumours proven by histopathology received CT, including 18 with pleomorphic adenomas, 14 with Warthin's tumours and 19 with malignant tumours. Size and attenuation of parotid tumours were measured. Compared with 5-min attenuation, the 30-s and 90-s PEW $\left(\mathrm{PEW}_{30}\right.$, $\mathrm{PEW}_{90}$ ) and RPEW $\left(\mathrm{RPEW}_{30}, \mathrm{RPEW}_{90}\right.$ ) were calculated. Results There was a significant difference in $\mathrm{PEW}_{30}$, $\mathrm{RPEW}_{30}, \mathrm{PEW}_{90}$ and $\mathrm{RPEW}_{90}$ in the parotid neoplasms groups $(P<0.01)$, and statistical significance existed simultaneously in pleomorphic adenomas vs malignant tumours and Warthin's tumours vs malignant tumours according to $S N K-q$ test. The optimal diagnosis results of malignancy with $100 \%$ specificity $(32 / 32)$ was obtained by using a combina-
\end{abstract}

D. K. Su $\cdot$ D. Xie $\cdot$ L. D. Liu $\cdot$ X. N. Zhu

Department of Radiology, Oncology Hospital,

Guangxi Medical University,

Nanning, People's Republic of China 530021

W. Zhao

Department of Radiation,

Oncology Hospital Guangxi Medical University Nanning,

Guangxi, People's Republic of China 530021

Present Address:

G. Q. Jin $(\bowtie)$

Department of Radiology, Oncology Hospital,

Guangxi Medical University,

Nanning, People's Republic of China 530021

e-mail: jgq2011@126.com tion of the following criteria: $-70 \%>\mathrm{PEW}_{30}<36 \%,-30 \%>$ $\mathrm{PEW}_{30}<19 \%, \mathrm{PEW}_{90}>12 \%$, and the sensitivity (74\%) for diagnosis of malignancy was yield.

Conclusions Wash-out ratio may assist in differentiating the benign from malignant parotid gland tumours. Combining the percentage of enhanced wash-out ratios of CT protocols can yield diagnostic results for malignancy.

Keywords Low-dose CT parotid gland neoplasm . Dynamic contrast-enhanced CT. Percentage enhancement wash-out ratio

\section{Introduction}

Salivary gland neoplasms account for approximately $3 \%$ of all head and neck tumours. About $70 \%$ of all salivary gland neoplasms occur in the parotid gland [1,2]. Several studies [3-6] have investigated the value of computed tomography (CT) in distinguishing parotid gland tumours. The results of some documents have demonstrated that $\mathrm{CT}$ could identify patients with pleomorphic adenomas, Warthin's tumours and malignant tumours, and evaluate the extent of parotid gland tumours. Moreover, detailed information on signal intensity and wash-out ratio in parotid gland tumours by contrast-enhanced MRI has been reported for pleomorphic adenomas, Warthin's tumours, and malignant tumours [710]. To our knowledge, very few data on the percentage enhanced wash-out ratio in low-dose multiple-phasic CT among pleomorphic adenomas, Warthin's tumours and malignant tumours have already been analysed. Consequently, this study focused on strategies to differentiate benign from malignant neoplasms by measuring the percentage enhanced washout ratio of patients with parotid gland tumours. 


\section{Materials and methods}

\section{Study cohort}

This study was approved by our institutional review board, and informed patient consent was obtained before study participation according to institutional and native guidelines. Between August 2002 and December 2009, the 51 patients (29 men and 22 women aged 25-68 years; mean age, $44 \pm 8.5$ years) fulfilled the criteria for inclusion. 18 patients (13 men and 5 women aged 26-58 years; mean age, $41 \pm 7.4$ years) had pleomorphic adenomas, and 14

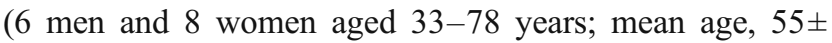
9.1 years) had Warthin's tumours. 19 patients (11 men and 8 women aged $25-68$ years; mean age, $46 \pm 8.6$ years) had malignancies, including adenoid cystic carcinoma $(n=6)$, mucoepidermoid carcinoma $(n=4)$, carcinoma ex pleomorphic adenoma $(n=2)$, ductal carcinoma $(n=2)$, acinic cell carcinoma $(n=1)$, sebaceous carcinoma $(n=1)$, lymphoma $(n=2)$ and squamous carcinoma $(n=1)$. Diagnoses for 51 patients with parotid gland neoplasms were established when histological proof was obtained at surgery.

\section{Imaging protocol}

All patients underwent multi-detector CT (LightSpeed ultra; GE Medical Systems, USA). All patients gave written informed consent to undergo CT. To reduce the radiation doses and preserve the diagnostic quality of the imaging, the protocol for the helical CT examinations performed in 51 patients with acquisition parameters: $5-\mathrm{mm}$ slice, a $7.5 \mathrm{~mm} / \mathrm{s}$ table speed, $120 \mathrm{kVp}, 60 \mathrm{~mA}$, a 0.8 -s rotation time, a pitch of 0.625 , and $5-\mathrm{mm}$ reconstruction intervals before and after the intravenous bolus injection of contrast material (Ultravist 300, Schering, Germany; $300 \mathrm{mg}$ of iodine per millilitre). Imaging began at the skull base and continued to the thyroid cartilage. To optimise the reproducibility of the starting measurements, each image was obtained while the patient held their breath and stopped deglutition.

First, a non-enhanced image was obtained through the parotid gland. An 18- or 20-gauge intravenous catheter was placed in an antecubital vein and manually tested by rapidly infusing $10 \mathrm{ml}$ of saline. Subsequently, $80-120 \mathrm{ml}$ of nonionic contrast material $(1.5 \mathrm{ml}$ per $\mathrm{kg})$ was administered at $2 \mathrm{ml} / \mathrm{s}$ by using a power injector (Medrad, Warrendale, PA, USA). In all patients, the second, third, and fourth images were obtained $30 \mathrm{~s}, 90 \mathrm{~s}$, and $5 \mathrm{~min}$ after the start of the contrast material injection respectively. The imaging protocol was preprogrammed so that these images were obtained by using the same parameters that were used to obtain the non-enhanced images. The images were obtained with standard soft-tissue settings (window width, $300 \mathrm{HU}$; window level, $40 \mathrm{HU}$ ).
Image and data analyses

The CT were interpreted and values of diagnostic parameters were measured by using Advantage Windows 4.2 (GE Medical systems, USA). These tasks were performed by two radiologists who were experienced in performing $\mathrm{CT}$ of the parotid glands. Two radiologists had no knowledge of the clinical or histological findings, and they worked independently without consultation with one another.

To determine the size of the masses, a distance cursor was used to measure the diameter in the transverse plane. The section of the largest square area of the parotid gland mass was used for analysis. The measurements obtained by the two radiologists were averaged.

The attenuation values of all parotid gland masses detected at $\mathrm{CT}$ were measured by using circular region-ofinterest (ROI) cursors placed over the area of disease (the biggest mass was selected if multiple lesions were present). The ROI circle was made as large as possible with sufficient margin to avoid partial volume effects. Cystic, necrotic and haemorrhagic components, as well as calcifications of the parotid gland masses, were excluded if they were present. Necrosis was defined as a region (within the mass) with an attenuation value similar to that of water $(-20 \mathrm{HU} \sim 20 \mathrm{HU})$ on the non-enhanced CT. Calcification was defined as a region with an attenuation value greater than $120 \mathrm{HU}$ on non-enhanced CT. Attenuation values were recorded and averaged for final data analysis.

We calculated the following diagnostic parameters for all masses: the absolute percentage enhanced wash-out ratio $(\mathrm{PEW})$ was calculated as $\mathrm{PEW}_{30}=\left[1-\left(\mathrm{A}_{300}-\mathrm{A}_{\mathrm{N}}\right) /\right.$ $\left.\left(\mathrm{A}_{30}-\mathrm{A}_{\mathrm{N}}\right)\right] \cdot 100$ or $\mathrm{PEW}_{90}=\left[1-\left(\mathrm{A}_{300}-\mathrm{A}_{\mathrm{N}}\right) /\left(\mathrm{A}_{90}-\right.\right.$ $\left.\left.A_{N}\right)\right] \cdot 100$, and the relative percentage enhanced wash-out ratio $\left(\mathrm{RPEW}_{30}\right.$ or $\mathrm{RPEW}_{90}$ ) was calculated as follows: $\left[\left(\mathrm{A}_{30}-\mathrm{A}_{300}\right) / \mathrm{A}_{30}\right] \cdot 100$ or $\left[\left(\mathrm{A}_{90}-\mathrm{A}_{300}\right) / \mathrm{A}_{90}\right] \cdot 100$, where $\mathrm{A}_{\mathrm{N}}$ is the attenuation on non-enhanced $\mathrm{CT}, A_{30}$ or $A_{90}$ is 30 or 90 -s enhanced attenuation, and $A_{300}$ is 5 -min enhanced attenuation.

\section{Statistical analyses}

Statistical analyses were performed with software (SPSS 13.0; Chicago, IL, USA). Primary statistical analysis of the pooled data (mean \pm standard deviations) was performed with the analysis of variance (ANOVA) to determine mean differences in objective measurements of parotid gland tumour groups by helical CT, followed by StudentNewman-Keuls $-q(S N K-q)$ test for multiple comparisons. $P<0.01$ was considered to indicate statistical significance. Receiver operating characteristic (ROC) analysis was employed to investigate the differentiation capability of the diagnostic parameters value for distinguishing malignant from benign parotid tumours when significant differ- 
ences existed simultaneously in the malignant tumours versus pleomorphic adenoma groups and malignant tumour versus Warthin's tumour groups. The optimal threshold value, determined by the maximal Youden index defined as sensitivity plus specificity minus 1 , was selected and the area under the ROC (AUC) was calculated. Then, corresponding sensitivity and specificity were calculated for individual parameters. In addition, we examined whether the diagnostic accuracy could be improved by combining these individual diagnostic parameters.

\section{Results}

Mass size

The biggest range of pleomorphic adenoma diameters was from $0.8 \mathrm{~cm}$ to $8.1 \mathrm{~cm}$, compared with those of Warthin's tumours $(2.2-3.3 \mathrm{~cm})$ and malignant tumours $(1.9-4.5 \mathrm{~cm})$. There were no significant differences in mean diameters among the pleomorphic adenoma, Warthin's tumour and malignant tumour groups $(P=0.41)$.

Non-enhanced CT, dual contrast-enhanced CT and 5-minute delay contrast-enhanced $\mathrm{CT}$

The means and the ranges of attenuation of different groups of parotid gland tumours are listed in Table 1. There were no significant differences among the mean attenuations of pleomorphic adenomas, Warthin's tumours and malignant tumours on non-enhanced CT $(P=0.047)$ and the fourth $\mathrm{CT}$ $(P=0.21)$. Although significant differences were found in the attenuation of the pleomorphic adenomas, Warthin's tumours and malignant tumours groups $(P<0.01)$ on the second and the third CT, there were simultaneously no differences between pleomorphic adenomas vs malignant tumours and Warthin's tumours vs malignant tumours by SNK-q test.

Sixty-two masses in 51 patients were detected by the identical imaging protocol, including 18 pleomorphic adenomas $(n=18), 18$ Warthin's tumours $(n=14)$ and 26 malignant tumours $(n=19)$. Calcifications were found in two pleomorphic adenomas, but not in any of the Warthin's tumours or malignant tumours. 8 of the 14 Warthin's tumours, 6 of the 18 pleomorphic adenomas and 4 of the 19 malignant tumours had regions of necrosis.

\section{Diagnostic ratios}

Diagnostic ratios of parotid gland neoplasms are listed in Table 2. There were significant differences in $\mathrm{PEW}_{30}$ and $\mathrm{RPEW}_{30}$ among these parotid gland tumour groups $(P<$ 0.01 ). By using a threshold value of $-70 \% \mathrm{PEW}_{30}$ and $-30 \%$ $\mathrm{RPEW}_{30}$ between pleomorphic adenomas and malignant tumours, the sensitivity could reach up to $89 \%$ and $89 \%$ respectively, but the specificity was low for malignant tumours. At the threshold value of $36 \% \mathrm{PEW}_{30}$ and $19 \%$ RPEW $_{30}$ between Warthin's tumours and malignant tumours, the sensitivity-specificity ratios were $100 \%: 93 \%$ and 100\%:86\% respectively.

There were significant differences in $\mathrm{PEW}_{90}$ and $\mathrm{RPEW}_{90}$ among the three parotid gland tumour groups. Applying a threshold value of $12 \% \mathrm{PEW}_{90}$ and $4 \%$ $\mathrm{RPEW}_{90}$ between pleomorphic adenomas and malignant tumours, the ratios of sensitivity to specificity were $95 / 89 \%$ and $95 / 94 \%$. At the cut-off value of $31 \% \mathrm{PEW}_{90}$ between Warthin's tumours and malignant tumours, the ratio of sensitivity-specificity was $63 / 71 \%$. The optimal threshold values of RPEW 90 were not obtained between the Warthin's tumours and malignant tumours (Table 3).

To obtain $100 \%$ specificity and maximal sensitivity in malignant gland tumours by using the combination of $\mathrm{PEW}_{30}$, RPEW $_{30}$ and PEW $_{90}$ (Table 4), we obtained the threshold value criteria as follows: $-70 \%>\mathrm{PEW}_{30}<36 \%,-30 \%>$ $\mathrm{PEW}_{30}<19 \%, \mathrm{PEW}_{90}>12 \%$, and $74 \%$ sensitivity was yielded (Fig. 1a-e).

\section{Discussion}

Parotid gland masses are common: about $80 \%$ of parotid tumours are benign, and most of them, about $60 \%$, are

Table 1 CT parameters for each group and statistical results of ANOVA

\begin{tabular}{|c|c|c|c|c|}
\hline CT Parameters & Pleomorphic adenomas $(n=18)$ & Warthin's tumours $(n=14)$ & Malignant tumours $(n=19)$ & $\boldsymbol{P}$ value \\
\hline Non-enhanced attenuation (HU) & $37 \pm 6.3(28-46)$ & $42 \pm 5.6(33-49)$ & $38 \pm 5(32-48)$ & 0.047 \\
\hline 30 -s enhanced attenuation (HU) & $57 \pm 11(32-75)$ & $93 \pm 22(38-120)$ & $69 \pm 10(52-88)$ & $<0.01$ \\
\hline 90-s enhanced attenuation (HU) & $69 \pm 15(31-95)$ & $80 \pm 16(37-105)$ & $88 \pm 18(56-126)$ & $<0.01$ \\
\hline 5-min enhanced attenuation (HU) & $79 \pm 18(30-102)$ & $69 \pm 13(35-87)$ & $76 \pm 13(49-101)$ & 0.21 \\
\hline
\end{tabular}

Data are mean \pm standard deviations, with ranges in parentheses

The $P$ value levels are for comparison among pleomorphic adenomas, Warthin's tumours and malignant tumours groups by using the analysis of variance $(P<0.01)$ 
Table 2 Diagnostic ratios for each group and statistical results of ANOVA

\begin{tabular}{|c|c|c|c|c|}
\hline CT Parameters & Pleomorphic adenomas $(n=18)$ & Warthin's tumours $(n=14)$ & Malignant tumours $(n=19)$ & $\boldsymbol{P}$ value ${ }^{*}$ \\
\hline $\mathrm{PEW}_{30}(\%)$ & $-188 \pm 341(-1400-67)$ & $49 \pm 11(29-75)$ & $-30 \pm 46(-121-33)$ & $<0.01$ \\
\hline $\mathrm{RPEW}_{30}(\%)$ & $-38 \pm 32(-93-18)$ & $24 \pm 8(8-39)$ & $-11 \pm 18(-51-18)$ & $<0.01$ \\
\hline $\mathrm{PEW}_{90}(\%)$ & $-39 \pm 98(-411-50)$ & $32 \pm 13(12-67)$ & $26 \pm 10(3-47)$ & $<0.01$ \\
\hline $\mathrm{RPEW}_{90}(\%)$ & $-14 \pm 21(-80-9)$ & $13 \pm 5(5-20)$ & $14 \pm 1(1-24)$ & $<0.01$ \\
\hline
\end{tabular}

Data are mean \pm standard deviations, with ranges in parentheses

The $P$ value levels are for comparison among pleomorphic adenomas, Warthin's tumours, and malignant tumours groups by using the analysis of variance $(P<0.01)$

pleomorphic adenoma, followed by Warthin's tumour (about 10\%). Malignant tumours comprise about 15 to $30 \%$ of all kinds of parotid tumours. The most common parotid gland malignancy is mucoepidermoid carcinoma [11]. The cystic adenoid carcinoma is the second most frequent malignancy; other parotid tumours include acinar cell carcinomas, adenocarcinoma not otherwise specified (NOS), lymphoma, and the carcinoma ex pleomorphic adenoma [12].

The main symptom in patients with parotid neoplasms is a lump in the parotid gland area. Other symptoms such as pain, facial palsy and skin ulcers may emerge in malignancies. According to the extent of the tumour, partial or total parotidectomy is the mainstream treatment measure for benign and malignant parotid tumours. Radiotherapy may aid in malignant cases as adjuvant therapy; chemotherapy is rarely applied. Parotid gland neoplasm recurrence rates of local, regional and distant are $40 \%, 15 \%$ and $11 \%$, respectively. Patients with recurrence have a poor prognosis [13].

The use of CT and MR imaging has resulted in the detection of parotid gland masses. More recent literature $[3-10,14,15]$ has focused on different approaches to characterising parotid masses, including assessing lesion size, describing morphological criteria, measuring attenuation or signal intensity, calculating the contrast material change of single or dual enhancement. Kress et al. [14] have demonstrated that CT sialography is dispensable in the diagnosis of suspected malignant tumours. Casselman and Mancuso [15] reported that $\mathrm{CT}$ and MR imaging were the first choices for providing the same diagnostic information in all cases of parotid gland neoplasms. Choi et al. [4] demonstrated that the ratio of CT numbers in enhancement patterns and the attenuation change was significantly different between Warthin's tumours and pleomorphic adenomas, and between Warthin's tumours and malignant tumours. Yabuuchi et al. [7] showed that parotid neoplasms could be differentiated by the $120 \mathrm{~s}$ of MR time of peak enhancement and a $30 \%$ wash-out ratio (WR), and benign and malignant tumours were recognised by a combined classification of time signal intensity curve and 30\% WR. However, to our knowledge, there are no reports of comparisons for benign and malignant parotid gland tumours by the attenuation and percentage enhancement wash-out ratios.

The results of our study indicated that the mean attenuation values of pleomorphic adenomas were similar to those of malignant tumours, but lower than those of Warthin's tumours on non-enhanced CT. However, there was considerable overlap in attenuation among pleomorphic adenomas, Warthin's tumours and malignant tumours, and, hence, there was no difference between benign and malignant parotid gland tumours $(P=0.047)$. In our limited data, multiple masses were found in patients with Warthin's tumours and malignant tumours. Necrotic portions were
Table 3 ROC analysis of percentage enhanced wash-out ratios of pleomorphic adenomas $(n=18)$, Warthin's tumours $(n=14)$, and malignant tumours $(n=19)$ of the parotid gland

\footnotetext{
${ }^{a}$ Data in parentheses are $95 \%$ confidence intervals

${ }^{\mathrm{b}}$ Sensitivity and specificity in the identification of malignant tumours. Data in parentheses are numbers used to calculate percentages
}

\begin{tabular}{lcccc}
\hline Parameters & AUC & Optimal cut-off value & Sensitivity (\%) & Specificity (\%) \\
\hline Differentiation between pleomorphic adenomas and malignant tumours & & \\
PEW $_{30}(\%)$ & $0.75(0.58-0.91)$ & -70 & $89(17 / 19)$ & $56(10 / 18)$ \\
RPEW $_{30}(\%)$ & $0.76(0.60-0.92)$ & -30 & $89(17 / 19)$ & $61(11 / 18)$ \\
PEW $_{90}(\%)$ & $0.92(0.82-1.00)$ & 12 & $95(18 / 19)$ & $89(16 / 18)$ \\
RPEW $_{90}(\%)$ & $0.98(0.94-1.00)$ & 4 & $95(18 / 19)$ & $94(17 / 18)$ \\
Differentiation between Warthin's tumours and malignant tumours $^{\mathrm{b}}$ & & \\
PEW $_{30}(\%)$ & $0.99(0.98-1.00)$ & 36 & $100(19 / 19)$ & $93(13 / 14)$ \\
RPEW $_{30}(\%)$ & $0.98(0.95-1.00)$ & 19 & $100(19 / 19)$ & $86(12 / 14)$ \\
PEW $_{90}(\%)$ & $0.64(0.45-0.84)$ & 31 & $63(12 / 19)$ & $71(10 / 14)$ \\
RPEW $_{90}(\%)$ & $0.47(0.27-0.68)$ & - & - & - \\
\hline
\end{tabular}


Table 4 Best diagnostic values for percentage enhanced wash-out ratios for differentiating benign from malignant tumours of the parotid gland

\begin{tabular}{lllr}
\hline Parameter & Threshold criterion & Parotid gland tumours & Specificity (\%) \\
\hline & & Sensitivity (\%) & $78(25 / 32)$ \\
$\operatorname{PEW}_{30}(\%)$ & $-70>$ and $<36$ & $89(17 / 19)$ & $69(22 / 32)$ \\
$\operatorname{RPEW}_{30}(\%)$ & $-30>$ and $<19$ & $89(17 / 19)$ & $72(23 / 32)$ \\
PEW $_{90}(\%)$ & $>12$ & $84(16 / 19)$ & $100(32 / 32)$ \\
Combined threshold values & -- & $74(14 / 19)$ & \\
\hline
\end{tabular}

Sensitivity and specificity in the identification of malignant tumours. Data in parentheses are numbers used to calculate percentages

viewed in three types of parotid tumours. Calcifications were detected in some pleomorphic adenomas, we presumed that the finding was related to ossification of cartilage structure in pleomorphic adenomas.

From our limited data, the mean attenuation of Warthin's tumour attained the peak of enhancement during 30-s imaging, and most malignant tumours attain peak attenuation in 90-s imaging, but significant differences did not exist simultaneously between the malignant tumour vs the pleomorphic adenoma groups and the malignant tumour vs the Warthin's tumour groups for a sustainable overlap. On the 5-min delay imaging, there was no difference among the parotid gland tumour groups. The discrepancy of mean attenuations between ours and a previous study [4] was attributed to CT parameters, such the total volume of contrast material and the injection rate of contrast material. Our findings suggested that reliable cut-off values were not helpful in differentiating benign from malignant tumours of the parotid gland on non-enhanced, 30-s, 90-s and 5-min imaging series.

The PEW and RPEW are the percentage enhanced washout ratio of enhanced attenuation according to the timing of the early and delayed imaging. Our results showed that there were significant differences in PEW and RPEW of parotid gland tumour groups, and significant differences simultaneously in the malignant tumour versus pleomorphic adenoma groups and the malignant tumour versus Warthin's tumour groups.

From 30-s to 5-min helical CT of parotid gland tumours, applying the threshold value of $-70 \%$ of $\mathrm{PEW}_{30}$ and $-30 \%$ of RPEW 30 between pleomorphic adenomas and malignant tumours, $89 \%$ and $89 \%$ of sensitivity, $56 \%$ and $61 \%$ of specificity were procured for the diagnosis of malignant tumours, relatively, $36 \%$ of $\mathrm{PEW}_{30}$ and $19 \%$ of $\mathrm{RPEW}_{30}$ between Warthin's tumours and malignant tumours, 93\% and $86 \%$ of specificity company with $100 \%$ sensitivity was diagnosed for malignancies. Likewise, from 90 -s to 5-min helical CT of parotid gland tumours, using an optimal cutoff value of $12 \%$ of $\mathrm{PEW}_{90}$ and $4 \%$ of $\mathrm{RPEW}_{90}$ between pleomorphic adenomas and malignant tumours, $95 \%$ and $95 \%$ of sensitivity, $89 \%$ and $94 \%$ of specificity were obtained for diagnosis of malignant tumours, and $31 \%$ of
PEW $_{90}$ between Warthin's tumours and malignant tumours, $63 \%$ of sensitivity and $71 \%$ of specificity were diagnosed for malignancies; conversely, with $\mathrm{RPEW}_{90}$ there was much more of an overlap between Warthin's tumours and malignant tumours.

Although, the enhanced wash-out ratio of tumours was altered for CT parameters [16], for instance, volume, injection rate and time of contrast material, the foundation of different enhanced wash-out ratios was determined by many inherent traits of parotid gland neoplasms, such as angiogenesis [17, 18]. With pleomorphic adenoma it is widely accepted that both epithelial and mesenchymal (myxoid, hyaline, chondroid, osseous) elements often arise from the same cell clone, which may be a myoepithelial or ductal reserve cell $[19,20]$. Warthin's tumours, also known as papillary cystadenoma lymphomatosum, consist of an epithelial, monomorphic, oncocytic component and a lymphoid stroma [21, 22]. Parotid gland malignancies include diverse tumour types, with the mucoepidermoid carcinoma being the most common tumour. The incidence of adenocarcinoma NOS, the acinar cell carcinoma, the cystic adenoid carcinoma, the carcinoma ex pleomorphic adenoma, and the undifferentiated carcinoma is obviously disparate in different research [23-27]. The enhancement wash-out ratio may mirror different histological components of tumour cells and different architecture of tumoral angiogenesis in parotid gland tumours. Hereby, the percentage-enhanced change could provide the pathological information and differentiate benign from malignant tumours in the parotid gland.

Although we set the optimal threshold criteria to obtain diagnostic values of individual percentage enhanced washout ratios, they had much more distinction with regard to sensitivity and specificity. We believe that identification of patients with malignant parotid tumours is more important than missing benign parotid tumours. For this reason, we reanalysed cut-off values of the individual percentage enhanced washout ratios to yield $100 \%$ specificity, and examined whether a combination of some of these parameters improved the sensitivity. We tried to employ one or two of three percentages enhanced changes to get $100 \%$ specificity, but failed. The final research demon- 
Fig. 1 a-e Transverse CT obtained in a 65 -year-old man with mucoepidermoid carcinoma. a Non-enhanced CT obtained at level of the right parotid gland shows ill-defined hypo-attenuation mass relative to the jugomaxillary muscle. 30 -s and 90-s contrast enhancement, 5-min delay (b, c, d) obtained at the same level as in a. The tumour has heterogeneous enhancement on the 30 -s (b), 90-s (c) and the 5-min delay phase (d) images. Tumour attenuation had $39.2 \mathrm{HU}$ in a, 68.4 HU in $\mathbf{b}, 76.7 \mathrm{HU}$ in $\mathbf{c}$, and 67.4 HU in d. Thus, the absolute percentage enhanced wash-out ratio of malignant tumours is $3.4 \%$ of $\mathrm{PEW}_{30}$ and $24.8 \%$ of $\mathrm{PEW}_{90}$, and the relative percentage enhanced wash-out ratio of the malignancy is $1.5 \%$ of RPEW30 and $12.1 \%$ of RPEW $_{90}$. e Surgical histopathology revealed mucoepidermoid carcinoma; $\mathrm{H} \& \mathrm{E} \times 100$
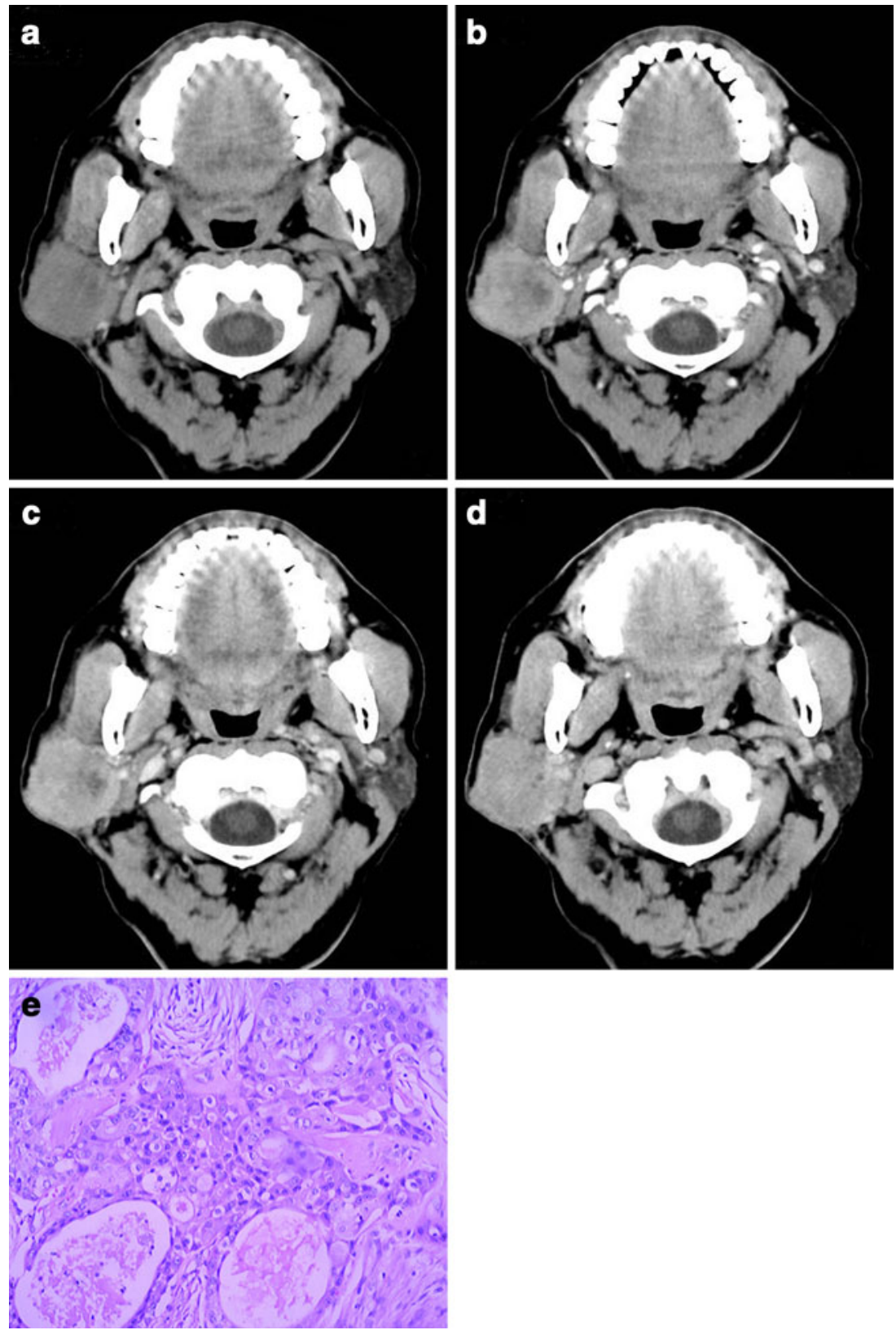

strated that a parotid gland tumour was defined as a malignant mass when the following parameters were satisfied: $\quad-70 \%>\mathrm{PEW}_{30}<36 \%, \quad-30 \%>\mathrm{PEW}_{30}<19 \%$, $\mathrm{PEW}_{90}>12 \%$, and $74 \%$ sensitivity.

Our study had some limitations. First, parotid gland malignancies include various types of tumours, such as mucoepidermoid carcinoma, adenoid cystic carcinoma, carcinoma ex pleomorphic adenoma, squamous cell carcinoma, acinic cell carcinoma, duct carcinoma and lymphoma. Second, some selected masses were biased in Warthin's tumours and malignant tumours, only the largest masses of the parotid gland were evaluated. Third, because of the small sample size and overlap in data among the three study subgroups, validation of the proposed cut-off values of CT diagnostic parameters needs to be performed in a larger scale trial. Lastly, if multiple-phasic CT were performed along with a diagnostic study, the patients would be exposed to an additional radiation dose.

In summary, the percentage wash-out ratios in contrast material-enhanced CT may reflect various characters of parotid gland neoplasms, and assist in differentiating the benign from malignant tumours. Combining the percentage enhanced wash-out ratios of the $\mathrm{CT}$ protocol can yield diagnostic results in malignant parotid gland tumours. 
Open Access This article is distributed under the terms of the Creative Commons Attribution Noncommercial License which permits any noncommercial use, distribution, and reproduction in any medium, provided the original author(s) and source are credited.

\section{References}

1. Nagler RM, Laufer D (1997) Tumors of the major and minor salivary glands: review of 25 years of experience. Anticancer Res 17:701-707

2. Pinkston JA, Cole P (1999) Incidence rates of salivary gland tumors: results from a population-based study. Otolaryngol Head Neck Surg 120:834-840

3. McGahan JP, Walter JP, Bernstein L (1984) Evaluation of the parotid gland. Comparison of sialography, non-contrast computed tomography, and CT sialography. Radiology 52:453-458

4. Choi DS, Na DG, Byun HS et al (2000) Salivary gland tumors: evaluation with two-phase helical CT. Radiology 214:231-236

5. Lev MH, Khanduja K, Morris PP et al (1988) Parotid pleomorphic adenomas: delayed CT enhancement. AJNR Am J Neuroradiol 19:1835-1839

6. Yerli H, Aydin E, Coskun M et al (2007) Dynamic multislice computed tomography findings for parotid gland tumors. J Comput Assist Tomogr 31:309-316

7. Yabuuchi H, Fukuya T, Tajima T et al (2003) Salivary gland tumors: diagnostic value of gadolinium-enhanced dynamic MR imaging with histopathologic correlation. Radiology 226:345-354

8. Ikeda M, Motoori K, Hanazawa T et al (2004) Warthin tumor of the parotid gland: diagnostic value of MR imaging with histopathologic correlation. AJNR Am J Neuroradiol 25:1256-1262

9. Alibek S, Zenk J, Bozzato A et al (2007) The value of dynamic MRI studies in parotid tumors. Acad Radiol 14:701-710

10. Okahara M, Kiyosue H, Hori Y et al (2003) Parotid tumors: MR imaging with pathological correlation. Eur Radiol 13:125-133

11. Spiro RH (1986) Salivary neoplasms: overview of a 35-year experience with 2,807 patients. Head Neck Surg 8:177-184

12. Yu GY, Ma DQ (1987) Carcinoma of the salivary gland: a clinicopathologic study of 405 cases. Semin Surg Oncol 3:240-244
13. Fitzpatrick PJ, Black KM (1985) Salivary gland tumors. J Otolaryngol 14:296-300

14. Kress E, Schulz HG, Neumann T (1993) Diagnosis of diseases of the large salivary glands of the head by ultrasound, sialography and CT-sialography. A comparison of methods. HNO 41:345-351

15. Casselman JW, Mancuso AA (1987) Major salivary gland masses: comparison of MR imaging and CT. Radiology 165:183-189

16. Jeong YJ, Lee KS, Jeong SY et al (2005) Solitary pulmory nodule: characterization with combined wash-in and washout features at dynamic multi-detector row CT. Radiology 237:67583

17. Passe TJ, Bluemke DA, Siegelman SS (1997) Tumor angiogenesis: tutorial on implications for imaging. Radiology 203:593-600

18. Wang JH, Min PQ, Wang PJ et al (2006) Dynamic CT evaluation of tumor vascularity in renal cell carcinoma. AJR Am J Roentgenol 186:1423-1430

19. Kondo T (2009) A case of lipomatous pleomorphic adenoma in the parotid gland. Diagn Pathol 4:16

20. Ide F, Kusama K (2004) Myxolipomatous pleomorphic adenoma: an unusual oral presentation. J Oral Pathol Med 33:53-55

21. Hatch RL, Shah S (2005) Warthin tumor: a common, benign tumor presenting as a highly suspicious mass. J Am Board Fam Pract 18:320-322

22. Russo C, Brunelli M, Baroli P (1993) Cytogenetic patterns in Warthin tumor. Acta Otorhinolaryngol 13:551-558

23. Wahlberg P, Anderson H, Biörklund A et al (2002) Carcinoma of the parotid and submandibular glands-a study of survival in 2465 patients. Oral Oncol 38:706-713

24. Goode RK, Auclair PL, Ellis GL (1998) Mucoepidermoid carcinoma of the major salivary glands: clinical and histopathologic analysis of 234 cases with evaluation of grading criteria. Cancer 82:1217-1224

25. Ostman J, Anneroth G, Gustafsson H (1997) Malignant salivary gland tumours in Sweden 1960-1989-an epidemiological study. Oral Oncol 33:169-176

26. Subhashraj K (2008) Salivary gland tumors: a single institution experience in India. Br J Oral Maxillofac Surg 46:635-638

27. Ito FA, Ito K, Vargas PA (2005) Salivary gland tumors in a Brazilian population: a retrospective study of 496 cases. Int J Oral Maxillofac Surg 34:533-536 